\title{
Impact of Arsenic Mitigation Program on Attitude of the Beneficiaries
}

\author{
Md. Safiul Islam Afrad \\ Dept. of Agricultural Extension and Rural Development, Bangabandhu Sheikh Mujibur Rahman \\ Agricultural University, Gazipur-1706, Bangladesh \\ Email: safiulislamafrad@yahoo.com
}

Received: 25 October 2010

Accepted: 17 March 2011

\begin{abstract}
The study was conducted to assess the impact of arsenic mitigation program on the attitude of the beneficiaries towards arsenic and its related issues in Bhanga and Matlab Upazila under Faridpur and Chandpur districts, respectively. Quantitative data were collected using a pre-designed interview schedule while qualitative data were collected by using focus group discussion and direct observation methods during January to March 2009. Findings revealed that 55\% of the patient respondents encountered medium to high changes in their attitude towards arsenic and the concerned issues. The differences of attitude of the patient and non-patient respondents between pre and post participation in arsenic mitigation programs were positively significant. It was also observed that education, farm size, organizational participation, contact with the sources of the information and annual family income had significant positive relationship with the change in attitude of the patient respondents. Again, education alone explained 69.4 percent of the total variations in attitude of the respondents, whereas contact with the sources of information explained 2.9 percent. It was recommended to arrange functional educational program focusing upon the rural poor to arsenic, sources of arsenic contamination, arsenicosis, arsenic removal measures and arsenic patient and appointment of efficient and enthusiastic health workers by the Public Health and Engineering Department for better result.
\end{abstract}

Keywords: Arsenic, attitude, beneficiaries

\section{Introduction}

Arsenic is a metalloid. Once it was lauded for its beneficial effects, but is now notorious for its toxicity and carcinogenicity. According to arsenic is a silent killer and undetectable in the early stages (Anon, 1984). Arsenic poisoning takes 8 to 14 years after ingestion to show its impact on health depending on the amount of arsenic ingested, nutritional status, and immune response of the individual. The social consequences of arsenic crisis are far-reaching and tragic. There has been little or no social education concerning the treatment of persons affected by arsenic poisoning. Poor literacy and lack of information often confuse the skin lesions with leprosy, which among village people is considered a contagious killer. As a result, those who have early symptoms of arsenicosis do not disclose their conditions to avoid certain ostracism. Affected school-age children are prevented from attending school and are avoided by their friends and classmates. Adults are barred from attending cultural and religious functions (Rahman, 2003).

Considering the severity of arsenic contamination in drinking water, different arsenic mitigation programs have been undertaken by the various Government and Nongovernment organizations (Anon, 2006). There are so far 25 organizations engaged in awareness raising in different parts of the arsenic contaminated areas at local and national levels working through a total of 60 projects and programs. The primary focus of those mitigation efforts in Bangladesh has been on the screening 
of arsenic contaminated tube-wells, identification of patient, treatment of the patients, provision of alternative water sources, increasing attitude of the affected people on arsenic and related issues (Anon, 2005).

It is, therefore, necessary to document the changes in attitude level of the beneficiaries towards arsenic and its related issues for smooth sailing of future programs regarding arsenic mitigation. This initiated the researcher to ask some relevant questions as: what is the attitude level of the beneficiaries of the arsenic mitigation program towards arsenic and related issues? And what are the relationships between the selected characteristics of the respondents and their changes in attitude? Considering the above circumstances, it was indispensable to investigate the change in attitude of the respondents due to their involvement in the arsenic mitigation program and to find out the relationship between the selected characteristics of the respondents and the changes in their attitude level due to participation in arsenic mitigation programs.

\section{Methodology}

\subsection{Locale of the study}

Faridpur and Chandpur districts were identified as the plause of devastating occurrence of arsenic contamination in ground water (Anon, 2004). Therefore, Bhanga and Matlab upazilas Faridpur and Chandpur districts, respectively were selected as the locale of the present study.

\subsection{Population and sampling}

The arsenic patients of the arsenic mitigation programs (UNICEF/DPHE fifteen upazila arsenic mitigation program and Arsenic in tubewell water and health consequences program) of the selected areas were considered as the target population of the present study. For smooth execution of sampling procedure the lists of the target population were collected from the authority of upazila sadar hospitals of Bhanga and Matlab. The number of sample population was 504 in Bhanga upazila and 250 in Matlab upazila that all together turned into 754. A size of 150 arsenic patients was selected as sample applying proportionate random technique. Another 50 non-patients were also selected for validation of results. Quantitative data were collected for the present study during January to March 2009 using a pre-designed interview schedule. Focus group discussion (FGD) and Direct observation were used to collect qualitative data for triangulation of results.

\subsection{Measurement of attitude}

Attitude of the respondent towards arsenic and related issues was measured by employing scale of 10 items related to arsenic, arsenicosis, arsenic removal measures and arsenic patient. The scale comprised of both positive and negative items. The item statements included in the scale were selected based on judges' rating and pre-test result following Edwards (1957). A 5-point Likert type scale was applied for each of the item-statement namely 'strongly agree', 'agree', 'undecided', 'disagree' and 'strongly disagree. In case of a positive item, score 05, 04, 03, 02 and 01 were assigned for the responses of 'strongly agree', 'agree', 'undecided', 'disagree' and 'strongly disagree', respectively. The reverse procedure was followed in assigning weightage for a response of negative item statements. Respondents' attitude about arsenic and related subject matter was measured both for before and after their participation in the mitigation programs. Thus, attitudinal score of a respondent could range from 10-50, where ' 10 ' indicates very unfavorable attitude and ' 50 ' indicates highly favorable attitude towards arsenic and related issues. The mean attitudinal value difference between before and after arsenic mitigation program was tested by administering t-test.

\subsection{Statistical analysis}

Spear Men correlation of coefficients was used to obtain relationships between selected characteristics of the respondents and their attitude change. Regression was employed in order to determine contribution of the selected characteristics on attitude change. Step wise regression analysis was also employed to 
individual contribution of the variables. The typical variation between the attitude of the patient respondents before and after their involvement with the arsenic mitigation programs was determined by testing the following null hypothesis: "there is no change in attitude of the patient respondents between their pre and post involvement situations with the arsenic mitigation programs".

\section{Results and Discussion}

\subsection{Attitude of the patient respondents}

The attitude of the patient respondents before their attachment with arsenic mitigation programs was arrayed from 12-45 against possible score range of 10-50 with an average of 26.43 (Table 1). The computed information revealed in that about three-fifths of the patient respondents $(61 \%)$ had highly favorable attitude, 30 percent had moderately favorable attitude although 9 percent of them were in low favorable attitude categories regarding arsenic and related subjects before their involvement with the arsenic mitigation programs. Thus, an overwhelming mass of the patient respondents (91\%) showed moderately to highly favorable attitude towards arsenic and related issues before their participation in the arsenic mitigation programs.

Table 1. Distribution of the patient respondents according to their attitude towards arsenic and related issues

\begin{tabular}{|c|c|c|c|c|c|c|c|c|c|}
\hline \multirow{3}{*}{ Categories } & \multicolumn{8}{|c|}{ Respondents } & \multirow{3}{*}{$\begin{array}{c}\text { Observed } \\
\text { "t" value } \\
\text { with df } \\
148\end{array}$} \\
\hline & \multicolumn{4}{|c|}{$\begin{array}{l}\text { Before involvement with } \\
\text { AMP* }\end{array}$} & \multicolumn{4}{|c|}{$\begin{array}{c}\text { After involvement with } \\
\text { AMP }\end{array}$} & \\
\hline & No & $\%$ & Mean & SD & No. & $\%$ & $\begin{array}{c}\text { Mea } \\
\mathrm{n}\end{array}$ & $\mathrm{SD}$ & \\
\hline Low favorable (up to 10 ) & 13 & 9 & \multirow{4}{*}{26.43} & \multirow{4}{*}{6.21} & - & - & \multirow{4}{*}{$\begin{array}{c}40.6 \\
6\end{array}$} & \multirow{4}{*}{6.36} & \multirow{4}{*}{$27.41 * *$} \\
\hline $\begin{array}{l}\text { Moderately favorable } \\
(11-15)\end{array}$ & 45 & 30 & & & 6 & 4 & & & \\
\hline $\begin{array}{l}\text { Highly favorable (15 \& } \\
\text { above) }\end{array}$ & 92 & 61 & & & 144 & 96 & & & \\
\hline Total & $\begin{array}{c}15 \\
0\end{array}$ & $\begin{array}{c}10 \\
0\end{array}$ & & & 150 & $\begin{array}{c}10 \\
0\end{array}$ & & & \\
\hline
\end{tabular}

*AMP= Arsenic Mitigation Program

Table 2. Distribution of the non-patient respondents according to their attitude towards arsenic and related issues

\begin{tabular}{|c|c|c|c|c|c|c|c|c|c|}
\hline \multirow{3}{*}{ Categories } & \multicolumn{8}{|c|}{ Respondents } & \multirow{3}{*}{$\begin{array}{c}\text { Observed " } \mathrm{t} \text { " } \\
\text { value with df } 48\end{array}$} \\
\hline & \multicolumn{4}{|c|}{$\begin{array}{l}\text { Before involvement with } \\
\text { AMP }\end{array}$} & \multicolumn{4}{|c|}{$\begin{array}{l}\text { After involvement with } \\
\text { AMP }\end{array}$} & \\
\hline & No. & $\%$ & Mean & SD & No. & $\%$ & Mean & SD & \\
\hline $\begin{array}{l}\text { Low favorable } \\
\text { (up to } 10 \text { ) }\end{array}$ & 48 & 96 & \multirow{4}{*}{26.38} & \multirow{4}{*}{6.51} & - & - & \multirow{4}{*}{41.88} & \multirow{4}{*}{5.27} & \multirow{4}{*}{$15.37 * *$} \\
\hline $\begin{array}{l}\text { Moderately } \\
\text { favorable (11-15) }\end{array}$ & - & - & & & - & - & & & \\
\hline $\begin{array}{l}\text { Highly favorable } \\
\text { (15 and above) }\end{array}$ & 2 & 4 & & & 50 & 100 & & & \\
\hline Total & 50 & 100 & & & 50 & 100 & & & \\
\hline
\end{tabular}


After attachment of the patient respondents in the arsenic mitigation programs, attitude was measured. The attitudinal score of the patient respondents were found from 22-50 against possible score range of 10-50 with an average of 40.66 (Table 1). Findings also indicate that 96 percent of them were fallen under highly favorable attitude group, whereas only 4 of them were found in moderately favorable attitude category after their participation in the mitigation programs. It is worthy of attention that none of the patient respondents fell under low favorable attitude category.

\subsubsection{Change in attitude of the patient respondents}

The attitude change of the patient respondents regarding arsenic and concerned subjects varied from 0-37 against the probable range of 0-40, with a typical of 12.49 . Fig. 1 reveals that a higher portion of the patient respondents $(45 \%)$ fell under the low attitude change category, while of 29 percent of them were under medium and 26 percent in high attitude change categories. Therefore, a great majority of the patient respondents (74\%) encountered low to medium attitude change regarding arsenic and related issues (Fig. 1).

\subsection{Attitude of the non-patient respondents}

The attitude score of the non-patient respondents before their involvement with arsenic mitigation programs ranged from 14-40, against possible score range of $10-50$ with an average of 26.38 and standard deviation of 6.51 (Table 2). Information showed that 96 percent non-patient respondents had low favorable attitude while only 4 percent showed highly favorable attitude regarding arsenic and related issues before their involvement with the arsenic mitigation programs. It is outstanding that none of them were under moderately favorable attitudinal category. Thus, a huge mass of the non-patient respondents (96\%) fell under low favorable attitudinal category and the figure was slightly lower in case of patient respondent, i.e., 91 percent of the patient respondents showed moderately to highly favorable attitudinal status at the same time (Table 1).

The attitude of the non-patient respondents after their involvement with arsenic mitigation programs ranged from 31-50 against possible score range of $10-50$ with an average of 41.88 (Table 2). Interesting information in Table 2 indicates that all of the non-patient respondents $(100 \%)$ had highly favorable attitude towards arsenic and related issues after their involvement in the arsenic mitigation programs. These findings were somewhat higher $(4 \%)$ than that of patient respondents at the same time (Table 1). This might happen so because the non-patient respondents might be afraid of the patient respondents' fatal sufferings and might be sincere to avoid such type of terrible situations.

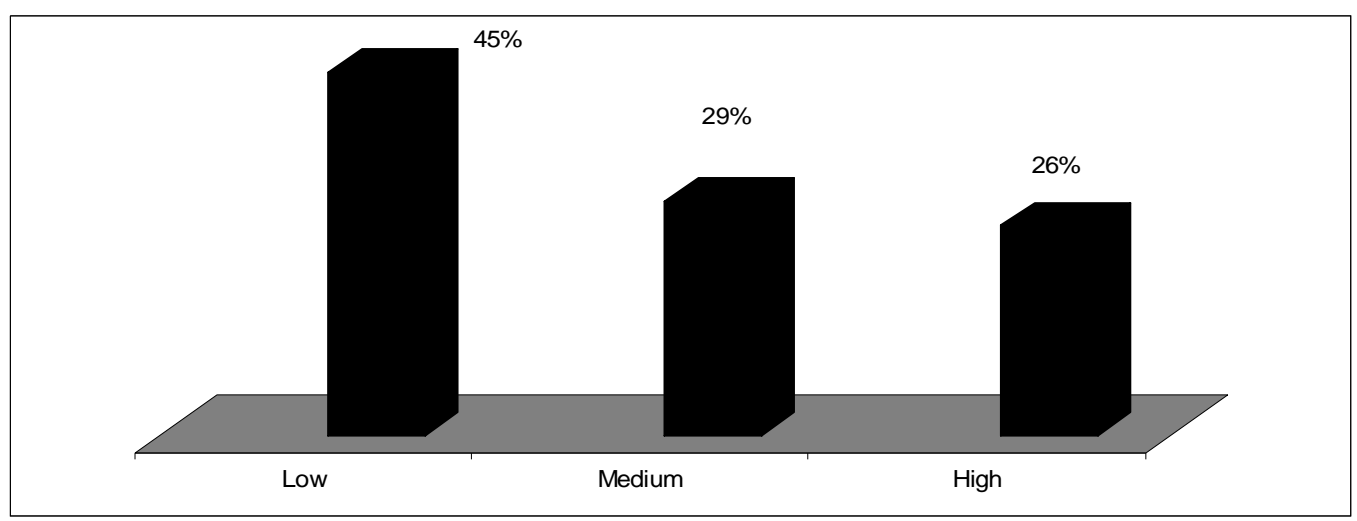

Fig. 1. Distribution of the patient respondents according to change in their attitude. 


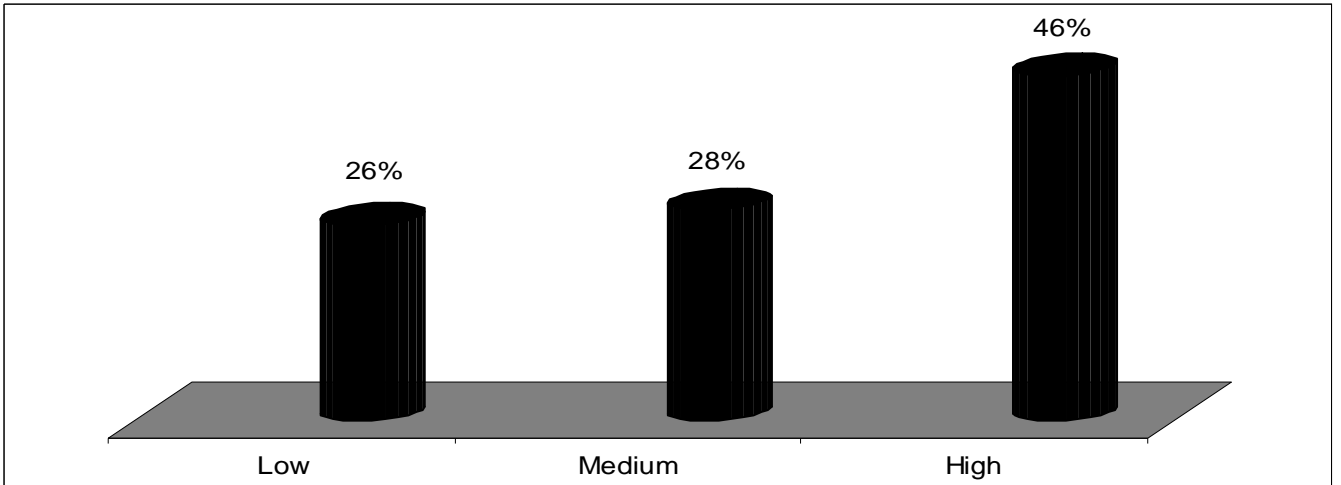

Fig. 2. Distribution of the non-patient respondents according to change in their attitude.

\subsubsection{Change in attitude of the non-patient respondents}

The attitude change of the non-patient respondents regarding arsenic and related issues varied from 2-31 against the possible range of 040 , with an average of 15.50 . Results furnished in the Fig. 2 reveal that 46 percent of the nonpatient respondents encountered high attitude change, 28 percent medium while 26 percent of them showed low attitude change. Thus, a greater majority of the non-patient respondents (74\%) had medium to high attitudinal change towards arsenic and related issues. Interestingly, the same portion (74\%) showed low to medium attitudinal change in case of patient respondents towards the same issue (Fig. 1).

It can be illustrated that attitude of the patient respondents changed significantly after their participation in the arsenic mitigation programs (Table 1). The patient respondents of both Bhanga and Matlab independently showed same findings. It can be explained that after their involvement in arsenic mitigation programs, the respondents knew the real information about the origin of arsenic and how it contaminated water and infected human body. They also received training from the programs' personnel. Thus, attitude change occurred within the patient respondents. In case of non-patient respondents, identical results were detected. In a study, Rosenboom (2004) found out 22 percent variations of attitude towards arsenic and related issues between follow-up and baseline survey.

\subsection{Direct observation}

Information presented in Box 1 indicates some important points, which should be taken into account in formulating any strategy focusing on arsenic issues for further improvement. From the observations in general, it became visible that respondents in both locations, changed their attitude towards arsenic and related issues to some extent. Though shortage of arsenic free tube-well within their vicinity, limited availability of handy reservoir for storing rain water came as vital constraint in getting arsenic free water, most of them still used arsenic free water. In particular, people in the studied areas have changed their attitude towards arsenic patients, arsenic mitigation options and development workers.

\subsection{Focus group discussion (FGD)}

The change in attitude of the beneficiaries towards arsenic patients and arsenic mitigation options occurred as they were taught with slide show, maintained frequent contact with health workers, attended group discussion with neighbors and local leaders etc. (Table 3 ). 


\section{Box 1. Recorded direct observation items \\ General observation:}

- Arsenic infection occurs irrespective of male and female

- People are aware of arsenic and related complicacy

- They behave gently with arsenic patients

- Arsenic patients claim that they do not have medicine from upazila hospital

- Most of them hardly manage to install arsenic free tube-well

- No significant initiatives are undertaken by local leaders in arsenic mitigation

- During rainy season, people don't have opportunity to produce vegetable as both of the locations are flood prone

\section{Observations related to attitude:}

- Villagers are found to have favorable attitude towards arsenic patients

- They let their neighbor to use arsenic free water source

- There is a strong belief that arsenic infection may divert into cancer

- Poor people find it curse from Almighty

Table 3. Change in attitude towards arsenic and related issues (Based on FGDs, $n=15+15=30$ )

\begin{tabular}{|c|c|c|}
\hline Areas & Extent of changes & Reasons for changes \\
\hline Attitude & Medium & $\begin{array}{l}\text { - } \text { Entertainment with slide show } \\
\text { - } \text { Frequent contact with health worker } \\
\text { - } \text { Group discussion with neighbors and local leaders } \\
\text { - } \text { Demonstration of flip chart }\end{array}$ \\
\hline
\end{tabular}

Table 4. Relationship between selected characteristics of the patient respondents and their attitude towards arsenic and related issues

\begin{tabular}{lc}
\hline Selected characteristics & Attitude \\
\hline Age & -0.075 \\
Education & $0.833^{* *}$ \\
Family size & -0.003 \\
Farm size & $0.265^{* *}$ \\
Organizational participation & $0.361^{* *}$ \\
Contact with the sources of information & $0.644^{* *}$ \\
Annual family income & $0.238^{* *}$ \\
Annual family expenditure & 0.157 \\
\hline
\end{tabular}

*Significant at $5 \%$ level, **Significant at $1 \%$ level 


\subsection{Relationship between attitude change and the selected characteristics}

A significant positive relationship was observed between educational qualification, farm size, organizational participation, contact with the sources of information and annual family income of the patient respondents with their attitude change (Table 4). The findings imply that with the increase in educational qualification, farm size, number of training received, organizational participation, and contact with the sources of information and annual family income of the patient respondents there would be an increase in their attitude towards arsenic and concerned subject matter. Chiang et al., (2010) in a study reported that continuing education program towards asthma patient care significantly increased the attitudes of pharmacists'.

\subsection{Contribution on change in attitude}

The multiple $\mathrm{R}$ and $\mathrm{R}^{2}$ values in the full model regression were 0.851 and 0.724 , respectively, which specified that all the six selected characteristics of the patient respondents explained 72.40 percent of the total variation of their attitudinal change towards arsenic and concerned subject (Table 5). However, only education and contact with the sources of information demonstrated positive significant role in the situation. The contribution of the other variables was insignificant. The trend was positive for farm size, annual family income and organizational participation in the situation.

Table 5. Multiple linear regression analysis of five selected characteristics showing their contribution on attitude change of the patient respondents

\begin{tabular}{lccc}
\hline Selected characteristics & $\begin{array}{c}\text { Un-standardized } \\
\text { coefficients }(\beta)\end{array}$ & $\begin{array}{c}\text { Standardized } \\
\text { coefficients }(\beta)\end{array}$ & t-value \\
\hline Education & 1.119 & .709 & $11.910^{* *}$ \\
Farm size & -.002 & .028 & .511 \\
Organizational participation & .056 & .005 & .105 \\
Contact with the sources of & -.009 & .207 & $3.616^{* *}$ \\
information & .001 & .001 & .019 \\
Annual family income & & 4.49 & \\
\hline Constant & & .724 & \\
$\mathrm{R}^{2}$ & & .851 & \\
Multiple R & & & \\
\hline
\end{tabular}

Table 6. Individual contribution of the selected characteristics of the patient respondents to change in attitude

\begin{tabular}{clccc}
\hline Step & \multicolumn{1}{c}{ Variable(s) added in each step } & $\mathrm{R}^{2}$ & $\mathrm{R}^{2}$ change & Explained variation \\
\hline 1 & Education & .694 & .694 & $69.40 \%$ \\
2 & $\begin{array}{l}\text { Education + Contact with the sources } \\
\text { of information }\end{array}$ & .723 & .029 & $69.40 \%+2.90 \%$ \\
\hline
\end{tabular}




\subsection{Contribution of education and sources of information on changing attitude}

The education and contact with the sources of information collectively explained the main fraction $(72.30 \%)$ of the total variations in attitude of the patient respondents regarding arsenic and related issues while only education elucidated 69.40 percent of the total variations (Table 6). On the other hand, 2.90 percent of total variation was explained for contact with the sources of information. It indicates that educational qualification and contact with the sources of information of the patient respondents exert remarkable influence on their attitude change towards arsenic and concerned issues.

\section{Conclusions}

Fifty five percent of the patient respondents encountered medium to high changes in their attitude regarding arsenic and the concerned issues. The attitudinal differences of the patient respondents between pre and post arsenic the mitigation programs situations were positively significant. Hence, it may be concluded that the arsenic mitigation programs have generated remarkable changes in attitude of the programs' beneficiaries towards arsenic and related issues. Education alone explained 69.40 percent total variations while contact with the sources of information explained 2.90 percent in change of attitude of the respondents. It may, therefore, be concluded that education is the key factor in changing attitude of the beneficiaries. Hence, the concerned authority should initiate massive educational program focusing upon the rural poor to arsenic, sources of arsenic contamination, arsenicosis, arsenic removal measures and arsenic patient with immediate effect. Besides, more visual teaching methods and aids should be used during motivational campaign. Efficient and enthusiastic health worker may be employed by the Public Health and Engineering Department (DPHE) for better result.

\section{References}

Anonymous. (Arsenic Policy Support Unit). 2005. The Response to Arsenic Contamination in Bangladesh: A Position Paper. Department of Public Health Engineering, Dhaka, Bangladesh.

Anonymous. (Bangladesh Arsenic Mitigation and Water Supply Project). 2004. Monitoring and Evaluation Services on Arsenic Awareness- raising During Screening Activities Being Implemented by BAMWSP in 147 Upazilas. Final Report.

Anonymous. 2002. (Who is Doing What on Arsenic Issues in Bangladesh).access in www.http;@yahoo.com. on 30 June 2006.

Anonymous. Ministry of Agriculture (MoA). 1984. Fisheries and Food. London: HM Stationary Office.

Chiang, Y. C., Lee, C. N., Lin, Y. M., Yen, Y. H. and Chen, H. Y. 2010. Impact of a continuing education program on pharmacists' knowledge and attitudes toward asthma patient care. Medical Principle and Practice, 19(4):305-11.

Edwards, A.L. 1957. Techniques of Attitude Scale Construction. New York: AppletonCentury-Crofts, Int.

Rahman, Z. 2003. Arsenic Poisoning: Bangladesh Faces a Disaster. A News Network Study. News Network.

Rosenboom, J.W. 2004. Not Just Red Or Green: An analysis of arsenic data from fifteen upazilas in Bangladesh. Arsenic Policy Support Unit and UNICEF. Department of Public Health Engineering, Dhaka, Bangladesh. 